NSF-ITP-94-21

March 1994

\title{
TOWARDS OBSERVING THE INTERCOMMUTATION OF FLUX TUBES IN SUPERCONDUCTORS
}

\author{
Ajit M. Srivastava \\ Institute for Theoretical Physics, University of California \\ Santa Barbara, California 93106, USA
}

\begin{abstract}
We propose a simple experiment to investigate the intercommutation of flux tubes in type II superconductors. Using this method the intercommutation of strings can be observed directly and the dependence of intercommutation on the angle of crossing of strings can also be analyzed.
\end{abstract}


Interaction of topological defects has been a subject of great interest. Interaction of flux tubes in type II superconductors is not only of theoretical interest but also has technological implications. In many particle theory models, strings in the early Universe play crucial role in generating density fluctuations responsible for the structure formation. In such models interaction properties of strings are of utmost importance. An essential property of cosmic strings is the intercommutativity; that is when two strings cross each other, they exchange partners at the crossing point as shown in Fig.1. Due to this property, large loops of cosmic strings chop themselves into smaller ones which rapidly decay through oscillations and emission of radiation. If this was not so then strings would have dominated the energy density of the Universe very early and would be observationally ruled out.

There have been many theoretical studies of string intercommutativity in relativistic field theory models. Shellard investigated string interactions numerically and calculated the probability of intercommutation as a function of the crossing angle and the relative velocity of strings for global U(1) strings [1]. He showed that strings almost always intercommute even for velocities very close to the speed of light. For gauge strings intercommutativity was demonstrated in numerical simulations by Moriarty, Myers and Rebbi [2]. Some qualitative understanding of the intercommutativity of global strings is provided in [包]. Intercommutativity for global strings has been seen to occur in liquid crystal experiments where one clearly sees strings chopping in smaller loops which shrink down rapidly [4]. Fluid vortices also show intercommutativity [5]. There is some indirect evidence for intercommutation of flux tubes in type II superconductors [6]. [Assuming that flux tubes intercommute, it has been suggested in [7] that the density of strings produced in a superconducting transition can be experimentally investigated.] However, a systematic experimental study of the intercommutation of strings has not been possible, primarily due to the difficulties in controlling the crossing of the strings.

In this letter we propose a simple experiment where the intercommutativity of flux tubes can be directly investigated. The essential idea of the experiment is that flux tubes in type II superconductors, which arise due to an external magnet, tend to follow the motion of the magnet if that magnet is gradually moved along the surface of the superconductor [8]. This provides us with a way of effectively holding the ends of the flux tubes. All that is needed after this is to take a crossed configuration of flux tubes and move magnets to force one bunch of flux tubes to go through the other bunch. Strings will then either intercommute or simply cross each other.

Let us describe the experiment in more detail now. Fig.2a shows the rectangular slab 
of superconducting material. As the mobility of strings is crucial in this case, high $\mathrm{T}_{c}$ materials are not suitable for this experiment due to vortex pinning. Even for conventional low $\mathrm{T}_{c}$ superconductors the sample should be very clean so vortex pinning is as little as possible. Crossed configuration of flux tubes is produced by two pairs of magnets. The first pair of magnets, $\mathrm{M}_{1}$ and $\mathrm{M}_{2}$, is aligned along the $\mathrm{x}$ axis so the flux tubes formed due to the field of these magnets are along the $\mathrm{x}$ axis. The second pair of magnets $\mathrm{M}_{3}$ and $\mathrm{M}_{4}$ is aligned along the $\mathrm{y}$ axis leading to strings along the $\mathrm{y}$ axis. $\mathrm{N}$ and $\mathrm{S}$ denote the north pole and the south pole respectively. These magnets need to have very small cross-sections so that each bundle of flux tubes is reasonably confined initially. The initial vertical separation of the two pairs of magnets along the $\mathrm{z}$ axis should be large compared to the spread of either bundle of flux tubes in $\mathrm{z}$ direction. Magnets are to be suspended so that their deflections can be monitored.

Now we fix the bottom pair of the magnets $\left(\mathrm{M}_{3}, \mathrm{M}_{4}\right)$ for simplicity and slowly move the top pair $\left(\mathrm{M}_{1}, \mathrm{M}_{2}\right)$ in the negative $\mathrm{z}$ direction. Let us call the distance between the $\mathrm{x}-\mathrm{y}$ planes going through the centers of the two magnet pairs as $h$. The forces on magnets have two components. One is $F_{\text {ext }}$, due to the magnetic field in the region outside of the superconductor and the second is $F_{s t r}$, due to trapped flux tubes [9]. As the vertical separation $h$ between the two pairs decreases, the two bundles of flux tubes will also approach each other. This movement of flux tubes due to the movement of the associated magnets is a reasonably natural thing to expect and there is also experimental evidence to support it [8]. [When magnet is moved considerably then associated string has to eventually move any way.]

When $h$ is decreased then, as long as the two bundles of strings are not touching each other, the only change in the force on the magnets is due to changes in $F_{\text {ext }}$, which is force due to the external field. This is because force between the two bundles is exponentially suppressed and as long as the two bundles of strings only get displaced and do not touch each other, $F_{\text {str }}$ should remain practically unchanged. As $h$ decreases, the magnitude of $F_{\text {ext }}$ will increase continuously as the two pairs of magnets are coming closer to each other and will reach maximum value for $h$ equal to zero, when all magnets are in the same plane. As $h$ is decreased further to negative values (i.e. the pair $\mathrm{M}_{1}, \mathrm{M}_{2}$ becomes lower than the pair $\mathrm{M}_{3}, \mathrm{M}_{4}$ ), the magnitude of $F_{\text {ext }}$ will start decreasing. This is not so with $F_{\text {str }}$ however. $F_{\text {str }}$ should remain unchanged initially as $h$ is decreased and should remain so until the two bundles of strings are about to touch. If the string bundles have roughly the same cross-section as that of the magnets then $F_{\text {str }}$ will remain unchanged until $h$ decreases to a value roughly equal to the diameter $d$ of the cross-section of a 
magnet. [Assuming that the magnets are moved slowly enough so that the strings follow the magnets. If the strings lag behind the magnets then $F_{\text {str }}$ may remain unchanged for even smaller values of $h$.]

As $h$ is decreased further, the two bundles will start strongly overlapping. Initially the two bundles may just repel each other for a little while meaning that $F_{\text {str }}$ will not change much. However, eventually some strings will break at the crossing point and will exchange partners, that is they will intercommute. These intercommuted strings will quickly shrink towards the edges of the sample and will lead to a drastic re-organization of flux tube distribution inside the sample. Fig.2b shows the situation when many strings have intercommuted and these strings are shrinking towards the edges of the sample. Some strings may simply cross each other without intercommuting as shown in Fig.2b. Thus there will be a critical value $h_{0}$ of $h$ at which the strings will start intercommuting. This should lead to a sudden change in the direction of $F_{s t r}$ as intercommuted strings shrink towards the edges. Since the net deflection of the magnets is a combined effect of $F_{\text {ext }}$ and $F_{\text {str }}$, a sudden change in $F_{\text {str }}$ should be observed as a sudden change in the positions of the magnets.

In fact, if the experiment is carried out extremely carefully then it may be possible to record the breaking of individual string (or at least very few strings at a time). For this one will need to consider very tiny cross-sections of the magnets and the magnetic field only slightly above the lower critical field $H_{c_{1}}$ so that the strings are very dilute in the bundle. Here $H_{c_{1}}$ is given by [10,

$$
H_{c_{1}}=\frac{\phi_{0}}{4 \pi \lambda^{2}} \ln \left(\frac{\lambda}{\xi}\right)
$$

where $\phi_{0}=\frac{h c}{2 e}$ is the flux quantum, $\lambda$ is the penetration depth and $\xi$ is the coherence length. From the relation between the induction $B$ and the magnetic field $H$ [10] we can choose large enough value of $H\left(\simeq 2 H_{c_{1}}\right)$ so that $B$ is of the order of $H_{c_{1}}$. Then the density of strings per unit surface area $n_{\text {ext }}$ due to the external magnets will be given by

$$
\begin{aligned}
n_{e x t} & =\frac{H_{c_{1}}}{\phi_{0}} \\
& =\frac{1}{4 \pi \lambda^{2}} \ln \left(\frac{\lambda}{\xi}\right)
\end{aligned}
$$

From this equation it is clear that by applying suitably weak external field, a dilute bundle of strings can be obtained where strings are well separated compared to the penetration depth. Fig. 3 shows a hypothetical plot of the deflection of magnets vs. $h$. This 
plot is intended to only show the qualitative aspects. As the variation of $F_{\text {ext }}$ with $h$ can be calculated knowing the positions of the magnets, the deflections $\theta_{\text {ext }}$ of the magnets due to only $F_{\text {ext }}$ can be subtracted out in plotting $\theta$ vs. $h$. Fig.3 is supposed to show such a normalized plot showing the deflection $\theta$ vs. $h$ entirely due to $F_{s t r}$. One way to get $\theta_{\text {ext }}$ due to $F_{\text {ext }}$ alone is by measuring the deflection vs. $h$ initially until the strings are about to touch. This gives the effect of $F_{\text {ext }}$ alone, and hence $\theta_{\text {ext }}$, for $h>h_{0}$ as $F_{\text {str }}$ is expected to remain unchanged for these values of $h$. Due to the symmetry of the setup, $\theta_{\text {ext }}$ will be symmetric about $h=0$. One may then get a rough idea of $\theta_{\text {ext }}$ for $0<h<h_{0}$ by using interpolation. Another possibility is to measure $\theta_{\text {ext }}$ for all values of $h$ using a slab of a different superconducting material, but same geometry, which has a larger value of $H_{c_{1}}$ so that no flux tubes are formed inside the superconductor for the magnets used.

As we mentioned earlier, until the two bundles of strings start touching, $F_{\text {str }}$ (and hence $\theta$ ) almost remains constant at some intial fixed value, as shown in Fig.3 for $h>d$ region of the plot. As $h$ is decreased further, strings will start overlapping. Sudden increase in $\theta$ for $h<h_{0}$ is supposed to represent breaking of one (or a small number of) strings. As $h$ decreases further, more and more strings break leading to further sudden changes in $\theta$. [Step in $\theta$ at a given $h$ will be governed by the number of strings which intercommute at that value of $h$.] It is important to realize that the jump in $\theta$ can in principle be very sharp. This is because $\theta$ will not change much if strings simply remain entangled. Breaking of string (and consequent intercommutation) is a discontinuous process and should lead to a discontinuous jump in $\theta$. If several strings intercommute at a time then departure from a sharp jump can arise due to the uncertainty in the number of strings breaking. Also, as the strings start breaking, they may affect the actual value of $F_{\text {ext }}$ leading to an error in properly subtracting the effects of $f_{\text {ext }}$ in plotting $\theta$ vs. $h$. This also can smoothen the jump in $\theta$. Here we would like to mention that if deflection due to $F_{\text {ext }}$ is not subtracted out in plotting $\theta$ vs. $h$, then the overall slope of the curve will be modified. However the steps in $\theta$ should still be present even if total deflection is plotted vs. $h$.

As $h$ is decreased further, eventually the two bundles will cross each other completely. A fraction of the strings will intercommute in this process leading to the maximum possible deflection $\theta_{m}$. This value $\theta_{m}$ gives us a measure of the final fraction of strings which have intercommuted and hence gives the probability of intercommutation. It is then a rather simple task to study the probability of intercommutation vs. the angle $\phi$ of crossing of strings. All that one needs to do is to change the direction of the alignment of the two magnets $M_{1}$ and $M_{2}$ so that the strings due to them make an angle $\phi$ with the strings formed by the pair $\mathrm{M}_{3}$ and $\mathrm{M}_{4}$. The whole experiment can be repeated then again leading 
to a plot of the deflection $\theta$ vs. $h$ (by properly subtracting the deflection due to $F_{\text {ext }}$ ). The value of the maximum deflection $\theta_{m}$ can then be noted down as a function of varying crossing angle $\phi$. This gives us a direct measure of the probability of intercommutation as a function of the crossing angle $\phi$. It should be clear that the value of $\phi$ for which $\mathrm{M}_{1}$ and $\mathrm{M}_{3}$ are on the same side of the superconducting slab (and $\mathrm{M}_{2}$ and $\mathrm{M}_{4}$ on the opposite side), the change in $F_{\text {str }}$ will be larger (as compared to the case shown in Fig.2) when the strings intercommute. This is because in that case the intercommuted strings will be able to completely shrink and get out of the sample. From this point of view, one may take a different geometry of the sample (such as hexagonal) so that intercommuted strings completely shrink away for other values of $\phi$ as well.

It is important to note that the above experiment should be carried out by moving magnets very slowly to guarantee that the strings are pulled along with the magnets. It is also of great interest to study the probability of intercommutation as a function of the relative velocity of the two strings. One can try to achieve this by moving down the magnet pair at different velocities keeping the crossing angle at some fixed value. This in principle can give the probability of intercommutation as a function of the velocity as well as the crossing angle. However, the velocity of strings through the superconductor is governed by many factors such as presence of impurities and dissipation. It will be hard in general to relate the velocity of magnet to the velocity of the flux tube in a direct manner. Still, the string velocity should certainly be an increasing function of the velocity of magnets. Therefore, qualitative features of the variation in the intercommutation probability with the relative velocity of the strings can be studied by following the procedure outlined in the above.

We conclude by pointing out the two crucial aspects of the experiment described in this letter. One is the ability to move flux tubes by the motion of the external magnet. This gives us a way of manipulating flux tubes almost as real strings. Secondly, the intercommutation of strings leads to a discontinuous rearrangement in the distribution of strings which can then be detected as a discontinuous change in the force on magnets. It may also be possible to use real time observation techniques employing Lorentz microscopy [11] to detect the rearrangement of strings after intercommutation.

\section{ACKNOWLEDGEMENTS}

I am very grateful to Mathew Fisher, Jim Langer, Fong Liu, Mike Stone and Shikha Varma for very useful Discussions. This work was supported by the U. S. National Science 
Foundation under Grant No. PHY89-04035.

\section{References}

[1] E.P.S. Shellard, Nucl. Phys. B283 (1987)624.

[2] K.J.M. Moriarty, E. Myers and C. Rebbi, Phys. Lett. B207 (1988)411.

[3] C. Rosenzweig and A.M. Srivastava, Phys. Rev. D43 (1991) 4029.

[4] M.J. Bowick, L. Chandar, E.A. Schiff and A.M. Srivastava, Science 263, 943 (1994);

I. Chuang, R. Durrer, N. Turok and B. Yurke, Science 251, 1336 (1991).

[5] J. Koplik and H. Levine, Phys. Rev. Lett. 71 (1993) 1375.

[6] M. G. Blamire and J. E. Evetts, Phys. Rev. B 33 (1986) 5131; M. A. R. LeBlanc, D. LeBlanc, A. Golebiowski and G. Fillion, Phys. Rev. Lett. 66 (1991) 3309; M. A. R. LeBlanc, S. Celebi, S. X. Wang and V. Plechacek, Phys. Rev. Lett. 71 (1993) 3367.

[7] S. Rudaz, A. M. Srivastava and S. Varma, preprint, ITP, University of California NSF-ITP-94-20/UMN-TH-1244/94.

[8] J. Pearl, Phys. Rev. Lett. 16 (1966) 99.

[9] R. J. Adler and W. W. Anderson, J. Appl. Phys. 68 (1990) 695.

[10] A.L. Fetter and P.C. Hohenberg in "Superconductivity", Vol. 2, Edited by R.D. Parks, (Marcel Dekker, Inc., New York, 1969).

[11] K. Harada, T. Matsuda, J. Bonevich, M. Igarashi, S. Kondo, G. Pozzi, U. Kawabe and A. Tonomura, Nature, 360 (1992) 51; K. Harada, T. Matsuda, H. Kasai, J. E. Bonevich, T. Yoshida, U. Kawabe and A. Tonomura, Phys. Rev. Lett. 71 (1993) 3371. 


\section{FIGURE CAPTIONS}

(1) Intercommutation of strings.

(2) (a) shows a rectangular slab of superconductor and the initial arrangement of the two pairs of magnets. The two bundles of strings are well separated initially along the $\mathrm{z}$ axis. (b) String distribution after the two bundles of strings have crossed leading to intercommutation of many strings. Ratio of strings which intercommuted to the ones that simply crossed gives a direct measure of the probability of intercommutation.

(3) This plot shows the qualitative aspects of the variation of the deflection $\theta$ vs. the vertical separation $h$ between the two magnet pairs. In this plot, it is assumed that the deflection due to the force on magnets by external magnetic field has been subtracted out. 

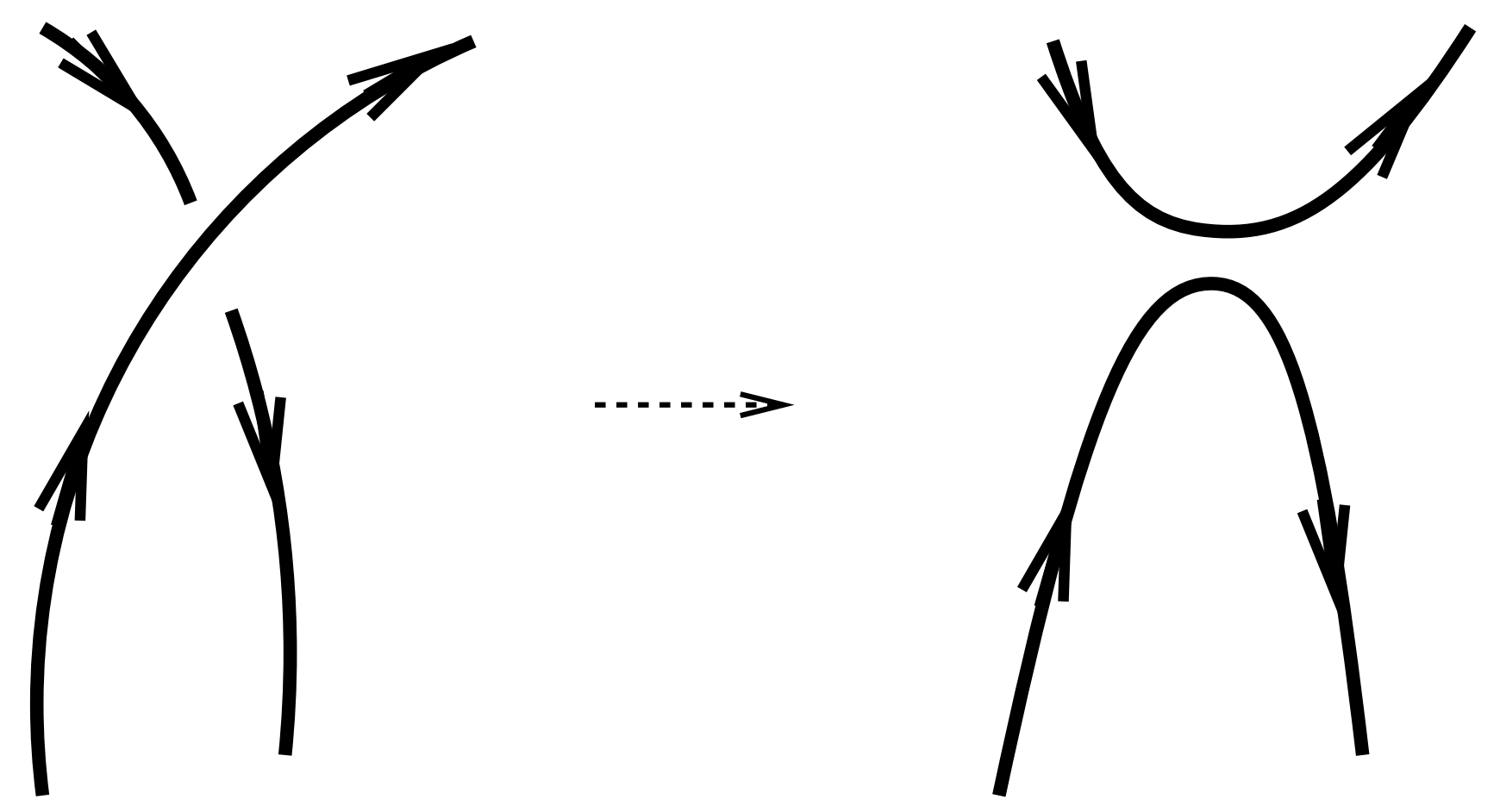

Fig. 1 

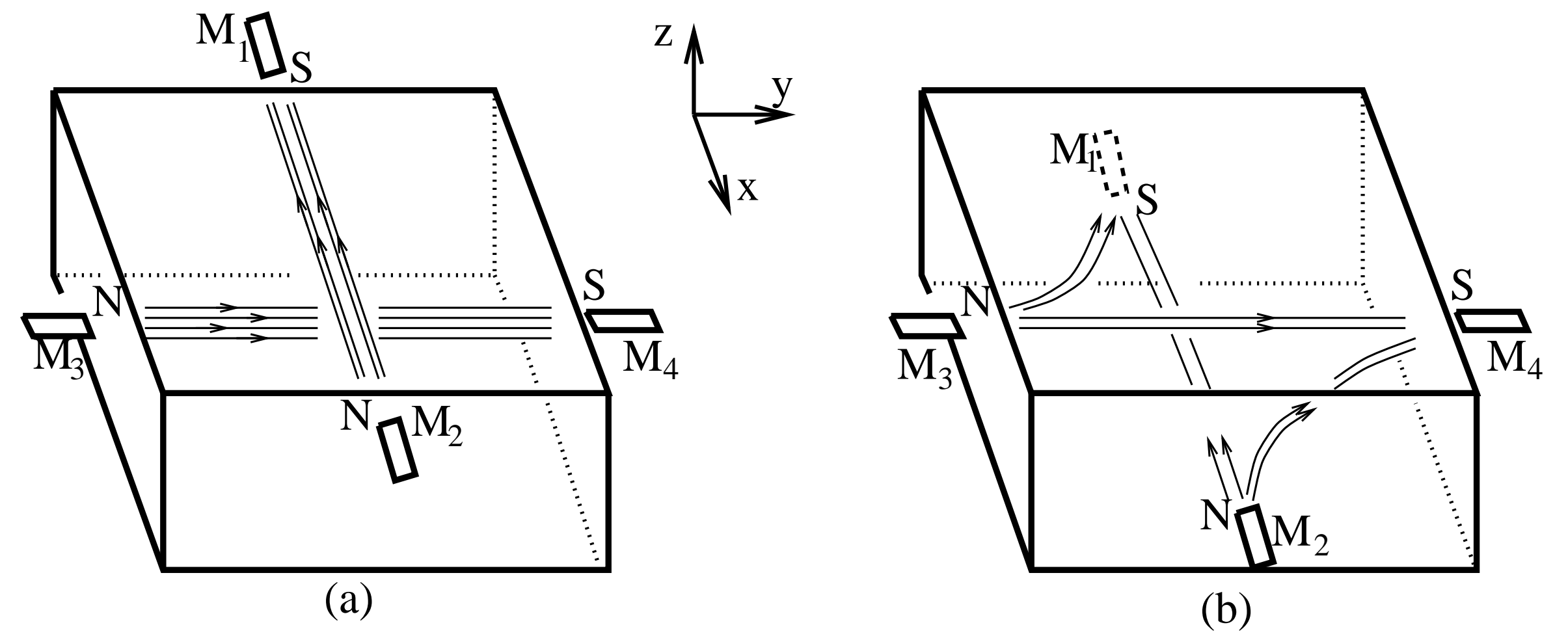

Fig. 2 


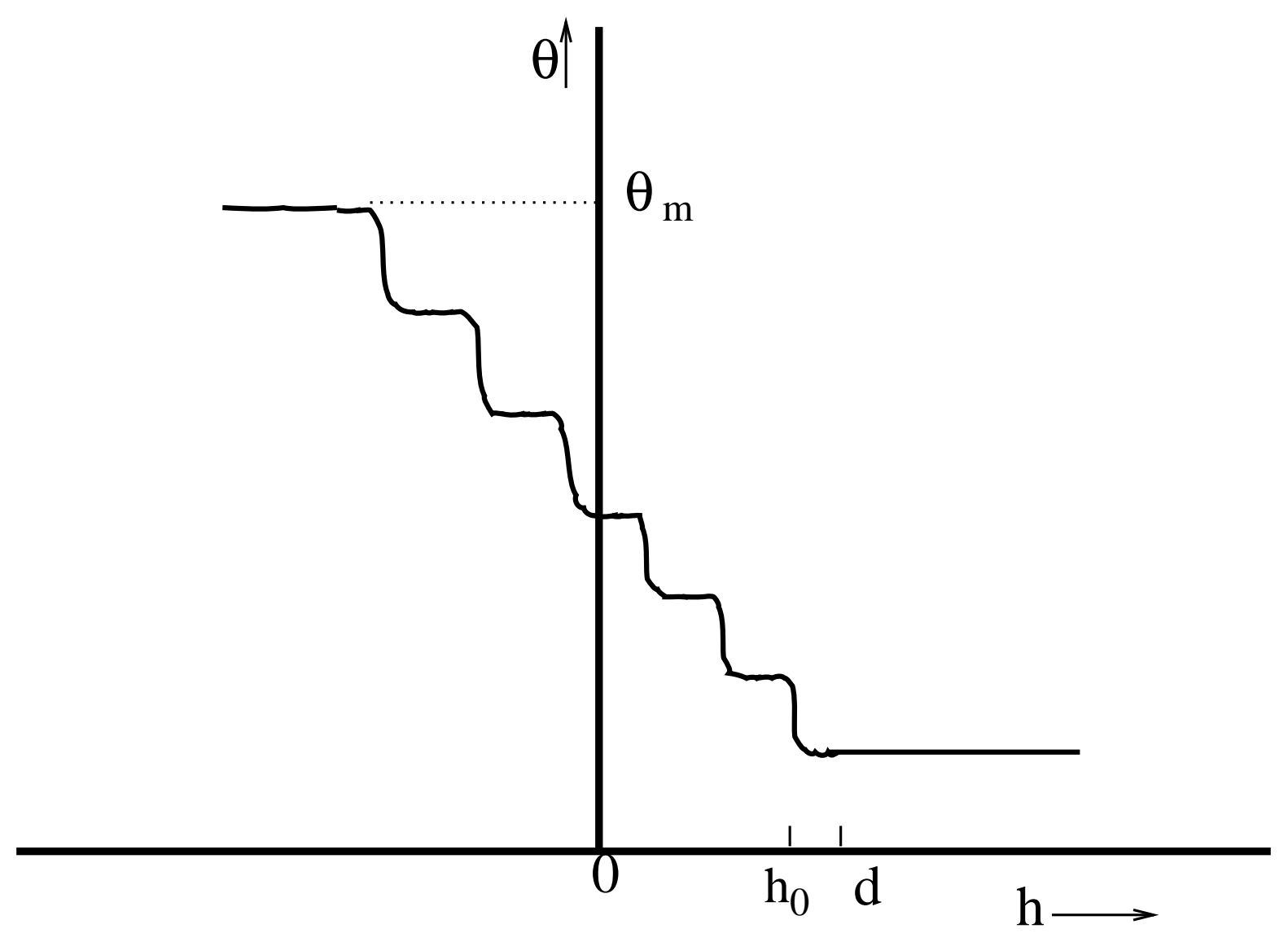

Fig. 3 